1 Supplementary Information for the manuscript:

2 Spatially asynchronous changes in strength and stability of terrestrial net 3 ecosystem productivity

$4 \quad$ Erqian Cui (eqcui@ stu.ecnu.edu.cn)

$5 \quad$ Chenyu Bian (cybian@stu.ecnu.edu.cn)

$6 \quad$ Yiqi Luo (yiqi.luo@nau.edu)

$7 \quad$ Shuli Niu (sniu@igsnrr.ac.cn)

$8 \quad$ Yingping Wang (Yingping.Wang@ @ csiro.au)

9 Jianyang Xia (jyxia@des.ecnu.edu.cn)

$10 \quad$ "To whom correspondence should be addressed. Email: jyxia@ des.ecnu.edu.cn

11 Supplementary figures

12 Figure S1 The micrometeorological tower sites with over 5 years of measurements in 13 the FLUXNET2015 Dataset.

14 Figure S2 Logarithm regression of annual NEP and $\frac{U}{R}$ for 72 FLUXNET sites.

Figure $\mathbf{S 3}$ The relationship between IAV in NEP (IAV NEP: quantified as the standard deviation of annual NEP) and $\ln \left(\frac{U}{R}\right)$.

Figure S4 The frequency distribution of ratio $\frac{\bar{U}}{\bar{R}}$ in this study.

\title{
Supplementary tables
}

Table S1 Summary of FLUXNET sites used in this study. 
Table S1. Summary of FLUXNET sites used in this study.

22

\begin{tabular}{|c|c|c|c|c|c|c|c|c|}
\hline Site & Lat $\left({ }^{\circ}\right)$ & Long $\left(^{\circ}\right)$ & Alt (m) & PFT & n (years) & Age & MATa $\left({ }^{\circ} \mathrm{C}\right)$ & $\operatorname{MAP}(\mathbf{m m})$ \\
\hline AT-Neu & 47.12 & 11.32 & 970 & GRA & 11 & & 6.79 & 669 \\
\hline AU-Dap & -14.06 & 131.32 & - & GRA & 7 & & 25.46 & 1403 \\
\hline AU-DaS & -14.16 & 131.39 & - & SAV & 7 & & 26.63 & 1452 \\
\hline AU-Dry & -15.26 & 132.37 & - & SAV & 7 & & 26.72 & 922 \\
\hline AU-Stp & -17.15 & 133.35 & - & GRA & 7 & & 26.07 & 761 \\
\hline AU-Tum & -35.66 & 148.15 & - & EBF & 14 & & 9.52 & 963 \\
\hline BE-Bra & 51.31 & 4.52 & 15 & $\mathrm{MF}$ & 9 & 78 & 10.84 & 831 \\
\hline BE-Lon & 50.55 & 4.75 & 167 & $\mathrm{CRO}$ & 11 & & 11.41 & 766 \\
\hline BE-Vie & 50.31 & 6.00 & 493 & $\mathrm{MF}$ & 19 & 75 & 8.35 & 965 \\
\hline CA-Man & 55.88 & -98.48 & 259 & ENF & 15 & 167 & -1.36 & 316 \\
\hline CA-Qfo & 49.69 & -74.34 & 382 & ENF & 8 & & 1.13 & 939 \\
\hline $\mathrm{CH}-\mathrm{Cha}$ & 47.21 & 8.41 & 393 & GRA & 10 & & 9.55 & 1144 \\
\hline $\mathrm{CH}-\mathrm{Dav}$ & 46.82 & 9.86 & 1639 & ENF & 18 & & 3.55 & 850 \\
\hline CH-Fru & 47.12 & 8.54 & 982 & GRA & 10 & & 7.72 & 1300 \\
\hline CH-Lae & 47.48 & 8.37 & 689 & $\mathrm{MF}$ & 11 & 100 & 7.82 & 1187 \\
\hline CH-Oe1 & 47.29 & 7.73 & 450 & GRA & 7 & & 9.27 & 1222 \\
\hline $\mathrm{CH}-\mathrm{Oe} 2$ & 47.29 & 7.73 & 452 & $\mathrm{CRO}$ & 11 & & 9.56 & 2063 \\
\hline CZ-wet & 49.02 & 14.77 & 426 & WET & 9 & & 8.40 & 609 \\
\hline DE-Akm & 53.87 & 13.68 & -1 & WET & 6 & & 9.23 & 648 \\
\hline DE-Geb & 51.10 & 10.91 & 161.5 & $\mathrm{CRO}$ & 14 & & 9.67 & 531 \\
\hline DE-Gri & 50.95 & 13.51 & 385 & GRA & 11 & & 8.58 & 943 \\
\hline DE-Hai & 51.08 & 10.45 & 430 & DBF & 13 & 138 & 8.33 & 761 \\
\hline DE-Kli & 50.89 & 13.52 & 478 & $\mathrm{CRO}$ & 11 & & 7.82 & 814 \\
\hline DE-Obe & 50.78 & 13.72 & 735 & ENF & 7 & & 6.49 & 1046 \\
\hline DE-Tha & 50.96 & 13.57 & 380 & ENF & 19 & 116 & 8.91 & 844 \\
\hline $\mathrm{DK}-\mathrm{NuF}$ & 64.13 & -51.39 & 50 & WET & 7 & & 0.13 & 954 \\
\hline DK-Sor & 55.49 & 11.64 & 40 & DBF & 19 & 86 & 8.40 & 861 \\
\hline DK-ZaH & 74.47 & -20.55 & 38 & GRA & 15 & & -8.84 & 161 \\
\hline FI-Hyy & 61.85 & 24.30 & 181 & ENF & 19 & & 4.41 & 611 \\
\hline FI-Sod & 67.36 & 26.64 & 180 & ENF & 14 & 100 & 0.75 & 531 \\
\hline FR-Fon & 48.48 & 2.78 & 103 & DBF & 10 & 150 & 11.43 & 691 \\
\hline FR-Gri & 48.84 & 1.95 & 125 & $\mathrm{CRO}$ & 10 & & 10.96 & 587 \\
\hline FR-LBr & 44.72 & -0.77 & 61 & ENF & 13 & 37 & 13.40 & 919 \\
\hline FR-Pue & 43.74 & 3.60 & 270 & $\mathrm{EBF}$ & 15 & 65 & 13.76 & 925 \\
\hline GF-Guy & 5.28 & -52.92 & 48 & EBF & 11 & & 25.61 & 3110 \\
\hline IT-BCi & 40.52 & 14.96 & 20 & $\mathrm{CRO}$ & 11 & & 18.06 & 1200 \\
\hline IT-Col & 41.85 & 13.59 & 1560 & DBF & 19 & 117 & 7.34 & 1159 \\
\hline IT-Cpz & 41.71 & 12.38 & 68 & $\mathrm{EBF}$ & 13 & & 15.40 & 820 \\
\hline IT-MBo & 46.01 & 11.05 & 1550 & GRA & 11 & & 5.21 & 974 \\
\hline IT-Noe & 40.61 & 8.15 & 28 & SHR & 11 & & 16.42 & 585 \\
\hline IT-Ro1 & 42.41 & 11.93 & 235 & DBF & 9 & 7.34 & 15.47 & 865 \\
\hline
\end{tabular}




\begin{tabular}{ccccccccc}
\hline IT-Ro2 & 42.39 & 11.92 & 160 & DBF & 11 & 16.37 & 15.45 & 786 \\
IT-SRo & 43.73 & 10.28 & 6 & ENF & 14 & 57 & 15.21 & 878 \\
IT-Tor & 45.84 & 7.58 & 2160 & GRA & 7 & & 2.97 & 776 \\
NL-Hor & 52.24 & 5.07 & 2.2 & GRA & 8 & & 10.60 & 1035 \\
NL-Loo & 52.17 & 5.74 & 25 & ENF & 18 & 90 & 10.07 & 865 \\
RU-Fyo & 56.46 & 32.92 & 265 & ENF & 17 & & 5.23 & 566 \\
US-ARM & 36.61 & -97.49 & 314 & CRO & 10 & & 15.27 & 646 \\
US-Blo & 38.90 & -120.63 & 1315 & ENF & 11 & 17 & 11.25 & 1401 \\
US-Cop & 38.09 & -109.39 & 1520 & GRA & 7 & & 13.62 & 203 \\
US-GLE & 41.37 & -106.24 & 3197 & ENF & 11 & 65 & -0.03 & 1405 \\
US-Ha1 & 42.54 & -72.17 & 340 & DBF & 22 & 84.5 & 8.16 & 1186 \\
US-Los & 46.08 & -89.98 & 480 & WET & 15 & 45 & 4.86 & 787 \\
US-Me2 & 44.45 & -121.56 & 1253 & ENF & 13 & 71 & 7.50 & 512 \\
US-MMS & 39.32 & -86.41 & 275 & DBF & 16 & 75 & 12.37 & 1083 \\
US-Ne1 & 41.17 & -96.48 & 361 & CRO & 13 & & 10.62 & 839 \\
US-Ne2 & 41.16 & -96.47 & 362 & CRO & 13 & & 10.34 & 861 \\
US-Ne3 & 41.18 & -96.44 & 363 & CRO & 13 & & 10.44 & 697 \\
US-NR1 & 40.03 & -105.55 & 3050 & ENF & 17 & 97 & 2.29 & 727 \\
US-PFa & 45.95 & -90.27 & 470 & MF & 20 & 39.5 & 5.74 & 606 \\
US-SRG & 31.79 & -110.83 & 1291 & GRA & 7 & & 18.77 & 388 \\
US-SRM & 31.82 & -110.87 & 1120 & SAV & 11 & & 19.01 & 333 \\
US-Syv & 46.24 & -89.35 & 540 & MF & 14 & 70 & 3.96 & 734 \\
US-Ton & 38.43 & -120.97 & 177 & SAV & 14 & & 16.33 & 555 \\
US-Twt & 38.11 & -121.65 & - & CRO & 6 & & 14.75 & 359 \\
US-UMB & 45.56 & -84.71 & 234 & DBF & 15 & & 7.16 & 613 \\
US-Umd & 45.56 & -84.70 & 239 & DBF & 8 & 90 & 7.09 & 738 \\
US-Var & 38.41 & -120.95 & 129 & GRA & 15 & & 15.87 & 563 \\
US-WCr & 45.81 & -90.08 & 520 & DBF & 16 & 80 & 5.63 & 732 \\
US-Whs & 31.74 & -110.05 & 1370 & SHR & 8 & & 17.58 & 284 \\
US-Wkg & 31.74 & -109.94 & 1531 & GRA & 11 & & 17.33 & 293 \\
ZA-Kru & -25.02 & 31.50 & 359 & SAV & 11 & & 21.88 & 449 \\
\hline Lat, & & & & & \\
UStitud & Long & & & & & \\
\end{tabular}




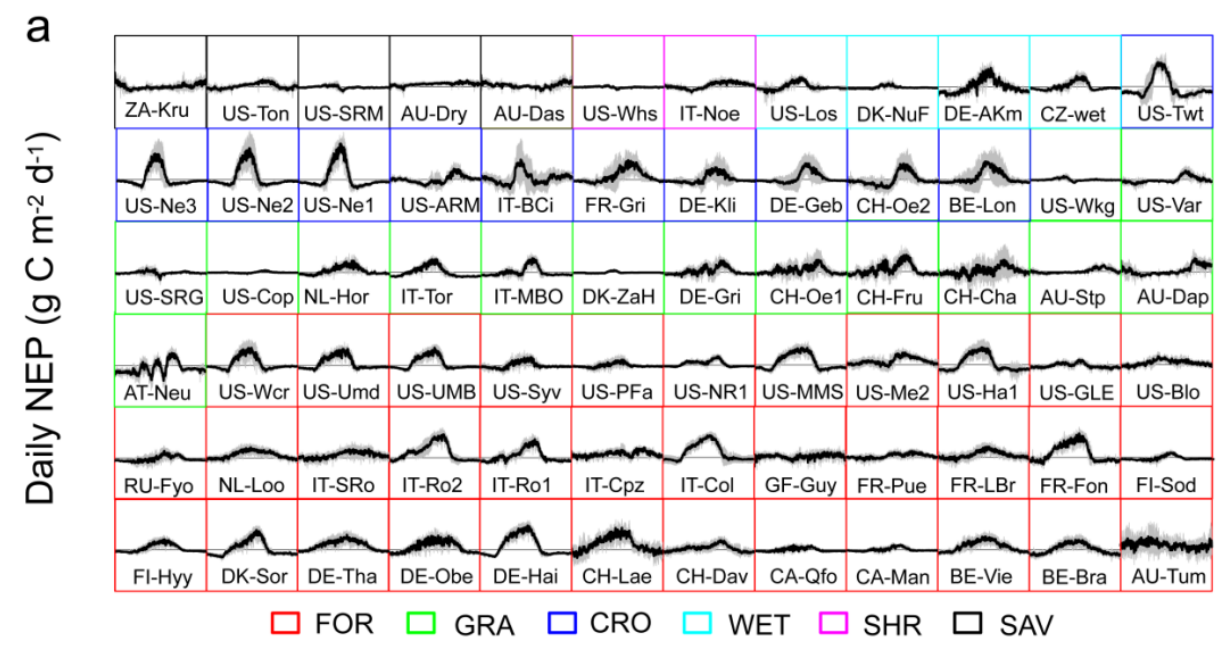

Day of year

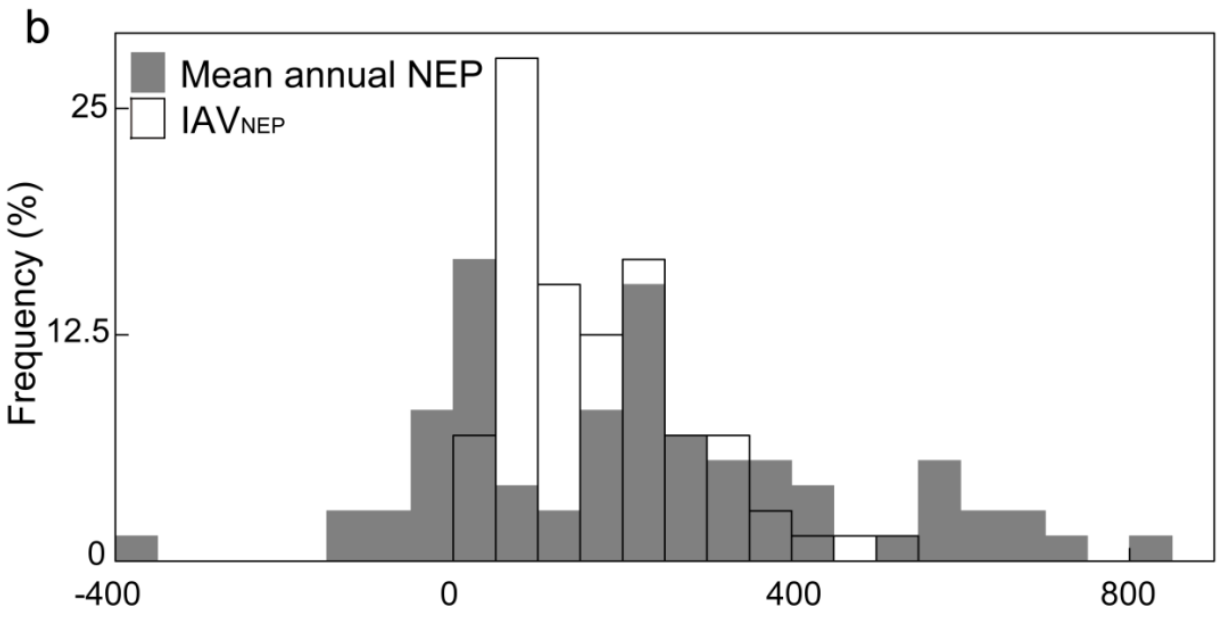

FLUXNET NEP $\left(\mathrm{g} \mathrm{C} \mathrm{m}^{-2} \mathrm{yr}^{-1}\right)$

32 Figure S1. The micrometeorological tower sites with over 5 years of measurements in the FLUXNET2015 Dataset. a, Interannual variation of terrestrial NEP among biomes. The solid lines represent the multi-year mean NEP and the shades show the interannual variation of annual NEP. b, Frequency distribution of mean annual NEP and interannual variability (IAV) of annual NEP (quantified as the standard deviation 37 of annual NEP). 


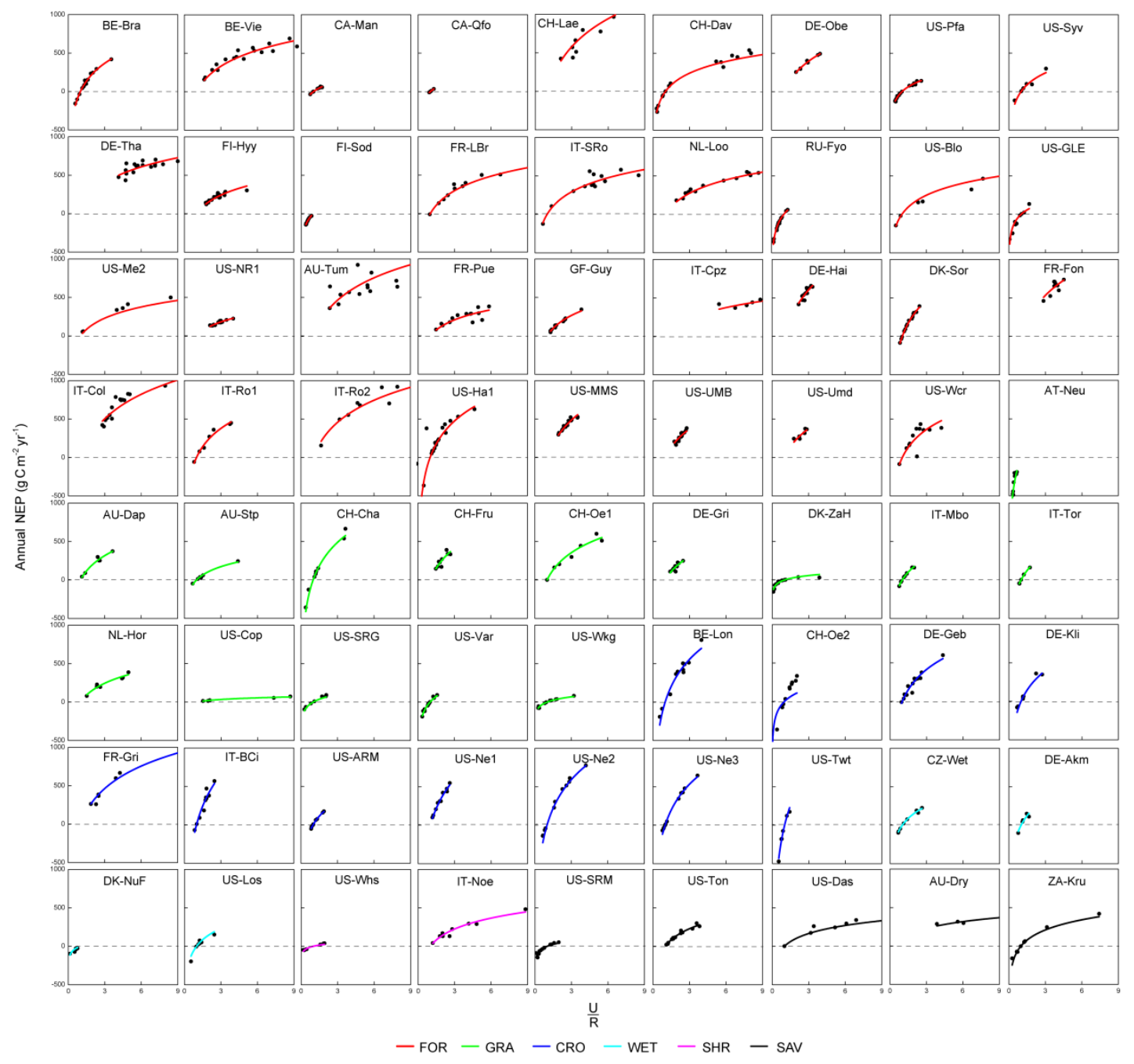

42 Figure S2. Logarithm regression of annual NEP and $\frac{U}{R}$ for 72 FLUXNET sites (of 43 the form NEP $=\beta \cdot \ln \left(\frac{U}{R}\right)$ ). Each site with at least 5 years of data. 


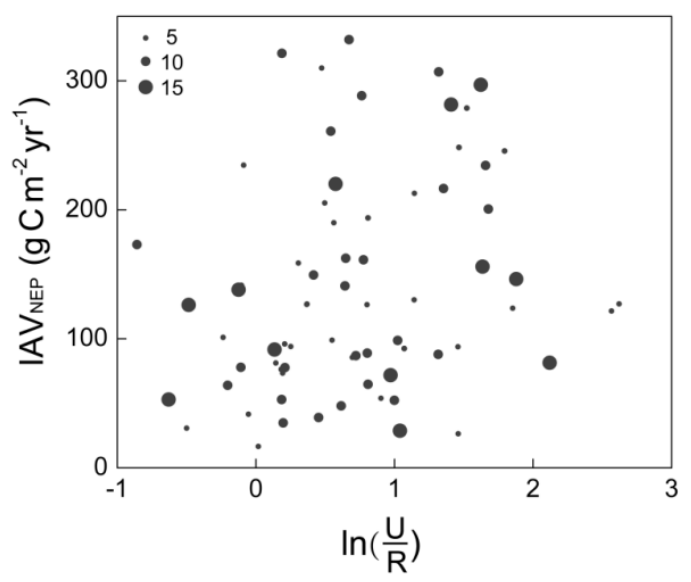

46

47 deviation of annual NEP) and $\ln \left(\frac{U}{R}\right)\left(R^{2}=0.048, P=0.052\right)$. The number of 48 49

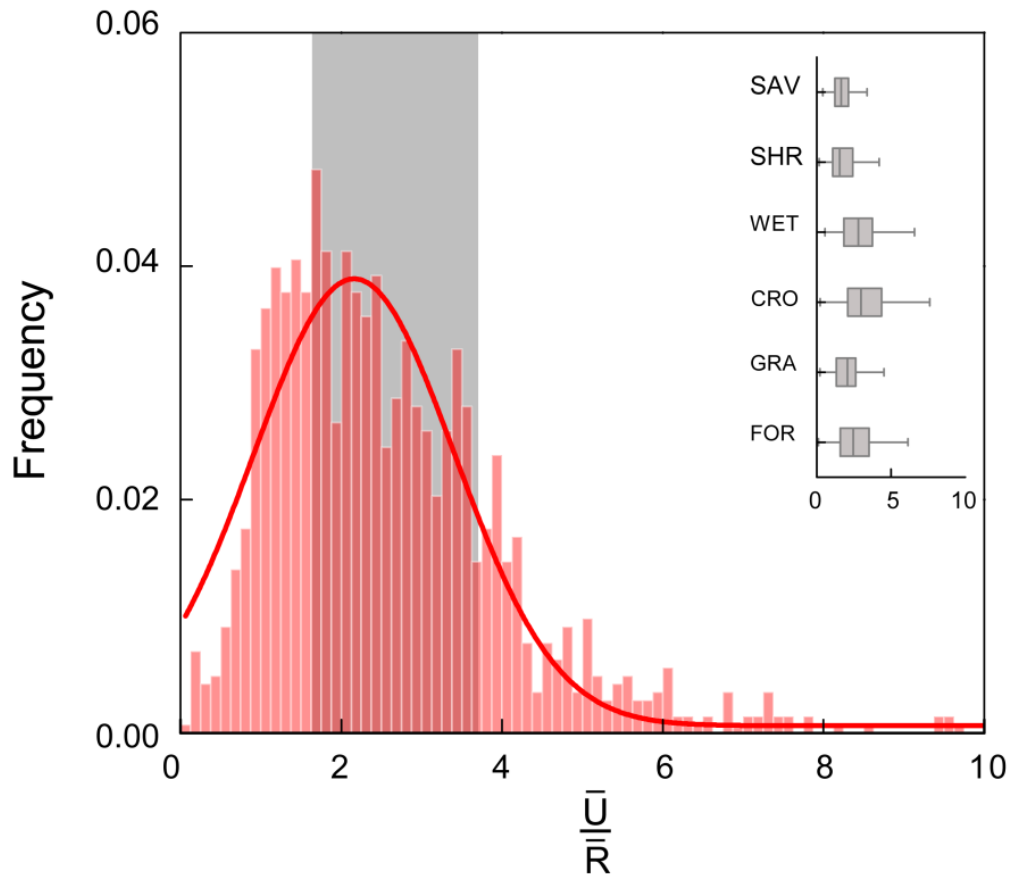

51 Figure S4. The frequency distribution of ratio $\frac{\bar{U}}{\bar{R}}$ in this study (red) and the range of 52 ratio $\frac{\bar{U}}{\bar{R}}$ reported by Churkina et al. (2005) (grey shading). The inset panel shows 53 distribution of $\frac{\bar{U}}{\bar{R}}$ over different plant functional types. 